\title{
The Canadian Immunization Monitoring Program, ACTive (IMPACT): Active surveillance for vaccine adverse events and vaccine-preventable diseases
}

\author{
Bettinger $\mathrm{JA}^{1^{*}}$, Halperin $\mathrm{SA}^{2}$, Vaudry $\mathrm{W}^{3}$, Law $\mathrm{BJ}^{4}$, Scheifele $\mathrm{DW}^{1}$, \\ on behalf of the Canadian IMPACT members
}

\author{
${ }^{1}$ Vaccine Evaluation Center, BC Children's Hospital and the University of British Columbia, Vancouver, BC \\ ${ }^{2}$ Canadian Center for Vaccinology, IWK Health Centre and Dalhousie University, Halifax, NS \\ ${ }^{3}$ Stollery Children's Hospital and University of Alberta, Edmonton, AB \\ ${ }^{4}$ Centre for Immunization and Respiratory Infectious Diseases, Public Health Agency of Canada, Ottawa, ON \\ "Corresponding author: jbettinger@cfri.ca
}

\begin{abstract}
For almost 25 years the Canadian Immunization Monitoring Program, ACTive (IMPACT) has been conducting active surveillance for severe adverse events following immunization (AEFIs) and vaccine-preventable diseases in children. The network, which consists of volunteer paediatric infectious diseases investigators at 12 tertiary care paediatric hospitals, is an important component of Canada's AEFI monitoring. The network employs nurses at each of the sites to search for and report possible AEFIs to local, provincial and national public health authorities. The active nature of the surveillance ensures a high level of vigilance for severe AEFIs in children.
\end{abstract}

\section{Introduction}

The Canadian Immunization Monitoring Program, ACTive (IMPACT) continues to be an innovative model for vaccine safety surveillance. 2014 marks its 23rd year of operation as a collaboration between the Canadian Paediatric Society and the Public Health Agency of Canada (PHAC). IMPACT active surveillance aims to detect unexpected or unusual occurrences that result in hospitalization after vaccination and to monitor vaccinepreventable diseases. It is well-positioned to detect and monitor changes in serious events, signals of concern, and emerging diseases in the paediatric inpatient population. This paper describes IMPACT's role in vaccine safety monitoring in Canada.

The original impetus for the project was increased public concern about vaccine safety, particularly around whole cell pertussis vaccines, and recognition of the need for enhanced vaccine safety surveillance in children after the passive surveillance system failed to identify an increased risk of aseptic meningitis from a new mumps combination vaccine in the mid-1980s $(1,2)$. IMPACT has two key active surveillance components: adverse events following immunization (AEFIs) and vaccine-preventable diseases. IMPACT's AEFI reporting reflects temporal associations, which means events are reported if they occur after a vaccination, but these events may not be caused by the vaccine. This follows the international best practice for AEFI reporting as causality cannot be determined a priori. IMPACT's vaccine-preventable disease surveillance monitors vaccine effectiveness by tracking children who continue to experience vaccine-preventable diseases.

IMPACT started in 1991 as a two-year pilot project in five tertiary care paediatric hospitals, expanded to 10 centres in 1993, to 11 in 1994, with the 12th centre added in 1999. Selection was based on geographical distribution, local centralization of pediatric beds, and availability of a pediatric infectious disease specialist to serve as the local IMPACT investigator. The current network encompasses approximately $90 \%$ of the pediatric tertiary care beds in Canada with about $50 \%$ of Canadian children directly residing within an IMPACT centre's catchment area. 
Because immunization history is not available at the time of admission for most hospitalized cases and because finding the immunization history for every hospital admission would be impractical, IMPACT uses predetermined targets as the first step to identify post-immunization adverse events (Table 1). These predetermined targets represent serious events for which either biologic plausibility exists that they could be caused by a vaccine or the severity of the event is such that immediate action would be warranted if a new signal were to be detected. The 12 IMPACT nurse monitors actively screen every hospital admission to determine whether or not it meets a predetermined target. All targets are then reviewed to determine whether or not there was a temporal association with previously administered vaccine(s). If an association is found (i.e., if a vaccine was administered within a specific time frame before the event) the event becomes an AEFI.

All AEFIs are reported within 15 days of being identified to PHAC for entry into the national pharmacovigilance database-Canadian Adverse Events Following Immunization Surveillance System (CAEFISS) —as well as to local or provincial public health officials to ensure appropriate follow-up at the individual level. Most target cases have no temporal association with a vaccine. Cases with an association, but with a confirmed non-vaccine cause, are not reported. However, if there is even a small doubt about the cause, the case is reported. In a typical year IMPACT monitors screen around 6,000 admissions to identify about 100 (less than 2\%) that are reportable as AEFIs. As a specific example, among cases screened from October 2013 to March 2014, a total of 3,084 target cases were identified and of these only 56 ( $1.8 \%$ of all identified) met the temporal criteria and were reported as an AEFI.

Table 1: The Canadian Immunization Monitoring Program ACTive (IMPACT) adverse events following immunization surveillance targets and reporting intervals, 2014

\begin{tabular}{|c|c|}
\hline Specific targets & IMPACT intervals for reporting \\
\hline \multicolumn{2}{|l|}{ Neurologic Events } \\
\hline Seizure & $\begin{array}{l}0-3 \text { days after inactivated vaccine(s); 0-15 days after live } \\
\text { vaccine }(\mathrm{s})^{1}\end{array}$ \\
\hline Guillain-Barré syndrome (GBS) & 0-42 days after inactivated or live vaccine(s) \\
\hline Other acute flaccid paralysis (AFP) & $0-42$ days after inactivated or live vaccine(s) \\
\hline Encephalitis & $0-42$ days after inactivated or live vaccine(s) \\
\hline Acute disseminated encephalomyelitis (ADEM) & $0-42$ days after inactivated or live vaccine(s) \\
\hline Myelitis & $0-42$ days after inactivated or live vaccine(s) \\
\hline Aseptic meningitis & $0-42$ days after inactivated or live vaccine(s) \\
\hline $\begin{array}{l}\text { Thrombocytopenia ( }<100 \times 10^{9} / \text { litre with clinical evidence of } \\
\text { bleeding, including Idiopathic Thrombocytopenic Purpura (ITP) }{ }^{2}\end{array}$ & $0-42$ days after inactivated or live vaccine(s) \\
\hline Intussusception in infants $<1$ year of age & Within 0-21 days after live attenuated rotavirus vaccine only \\
\hline $\begin{array}{l}\text { Vasculitides_(Kawasaki disease, Henoch-Schonlein Purpura (HSP), } \\
\text { etc.) }\end{array}$ & $0-42$ days after inactivated or live vaccine(s) \\
\hline \multicolumn{2}{|l|}{ Complication of vaccination } \\
\hline Anaphylactic shock & 48 hours after any vaccine \\
\hline Vaccination site cellulitis or abscess & $\begin{array}{l}\text { No specific timeline but needs to be localized to the } \\
\text { vaccination site. }\end{array}$ \\
\hline $\begin{array}{l}\text { Non-vaccination site infectious complication including sepsis or } \\
\text { infection of a normally sterile body site }\end{array}$ & $\begin{array}{l}\text { No specific timeline but needs clear evidence linking the } \\
\text { infection to a prior vaccination. }\end{array}$ \\
\hline $\begin{array}{l}\text { Varicella vaccine reactivation illness (Varicelliform rash or } \\
\text { Zosteriform rash) }\end{array}$ & $>42$ days after varicella vaccination \\
\hline $\begin{array}{l}\text { Other AEFIs: All reportable AEFIs that the monitor finds during } \\
\text { searches for the above IMPACT targets. }\end{array}$ & Follow the CAEFISS user guide \\
\hline
\end{tabular}


An AEFI detected after BCG (bacille Calmette-Guerin) vaccine is a good example of IMPACT in action. IMPACT's identification of deaths following BCG vaccination (3) prompted a review of BCG complications that indicated the risk of severe complications in First Nations infants was high and led to changes in the routine use of BCG vaccine in First Nations populations (4). The first evidence of improved safety with acellular pertussis vaccines was demonstrated with IMPACT data, which found a $79 \%$ decrease in febrile seizures associated with the receipt of pertussis vaccine (5). The data have been used to quantify the risk of hypotonic-hyporesponsive episodes and post-vaccination seizures in children $(6,7)$. IMPACT also published the largest case series in the world on postvaccination thrombocytopenia, showing that the condition is usually benign and resolves within one month in most children (8-10).

\section{Discussion}

IMPACT's focus on admitted paediatric cases means the most serious AEFI in children are actively sought and identified. Although less serious AEFI signals might not be identified through IMPACT, it fulfills a key role in Canada's vaccine safety surveillance and is an excellent complement to Canada's other AEFI reporting systems. Over the next three years the network will transition to electronic reporting, which will enable faster transmission and follow-up of information.

While IMPACT captures approximately $90 \%$ of pediatric tertiary care beds in Canada, it does not capture all paediatric admissions. With only two centres in Ontario, Canada's most populous province, the network does not adequately represent tertiary care admissions there. Solutions are being sought to address this. In spite of these limitations, IMPACT continues to be well suited to the Canadian context and provides a high level of vigilance for serious AEFIs among children.

\section{Acknowledgements}

Many thanks to Natalie Bridger, Scott Halperin, Karina Top, Roseline Thibeault, Dorothy Moore, Marc Lebel, Nicole Le Saux, Dat Tran, Joanne Embree, Ben Tan,Athena McConnell, Wendy Vaudry, Taj Jadavji, Otto Vanderkooi, David Scheifele, Laura Sauvé, and Julie Bettinger. We also gratefully acknowledge the expertise and contribution of the IMPACT nurse monitors, IMPACT Data Centre team and IMPACT coordinator.

\section{Conflict of interest}

None

\section{Funding}

The Canadian Immunization Monitoring Program, Active (IMPACT) is a national surveillance initiative managed by the Canadian Paediatric Society. Funding for AEFI surveillance activities is provided by the Public Health Agency of Canada. JAB is supported by a Career Investigator Award from the Michael Smith Foundation for Health Research.

\section{References}

(1) Scheifele DW. IMPACT after 17 years: Lessons learned about successful networking. Paediatr Child Health. 2009 Jan; 14(1):33-5.

(2) Scheifele DW, Halperin SA. Immunization Monitoring Program, Active: A model of active surveillance of vaccine safety. Semin Pediatr Infect Dis. 2003 Jul;14(3):213-9.

(3) Scheifele D, Law B, Jadavji T. Disseminated bacille Calmette-Guerin infection: Three recent Canadian cases. IMPACT. Immunization Monitoring Program, Active. CCDR. 1998 May 1; 24(9):69-72; discussion 3-5.

(4) Deeks SL, Clark M, Scheifele DW, Law BJ, Dawar M, Ahmadipour N, et al. Serious adverse events associated with bacille Calmette-Guerin vaccine in Canada. Pediatr Infect Dis J. 2005 Jun; 24(6):538-41. 
(5) Le Saux N, Barrowman NJ, Moore DL, Whiting S, Scheifele D, Halperin S. Decrease in hospital admissions for febrile seizures and reports of hypotonic-hyporesponsive episodes presenting to hospital emergency departments since switching to acellular pertussis vaccine in Canada: a report from IMPACT. Pediatrics. 2003 Nov; 112(5):e348.

(6) Top KA, Constantinescu CM, Laflèche J, Bettinger JA, Scheifele DW, Vaudry W, et al. Applicability of the Brighton Collaboration Case Definition for seizure after immunization in active and passive surveillance in Canada. Vaccine. 2013 Nov 19; 31(48):5700-5.

(7) Gold R, Scheifele D, Halperin S, Dery P, Law B, Lebel M, et al. Hypotonic-hyporesponsive episodes in children hospitalized at 10 Canadian pediatric tertiary-care centres, 1991-1994. CCDR. 1997 May 15; 23(10):73-6; discussion 6-8.

(8) Jadavji T, Scheifele D, Halperin S. Thrombocytopenia after immunization of Canadian children, 1992 to 2001. The Pediatric Infectious Disease Journal. 2003 Feb; 22(2):119-22.

(9) Sauvé LJ, Scheifele D. Do childhood vaccines cause thrombocytopenia? Paediatr Child Health. 2009 Jan; 14(1):31-2.

(10) Sauvé LJ, Bettinger J, Scheifele D, Halperin S, Vaudry W, Law B. Postvaccination thrombocytopenia in Canada. The Pediatric Infectious Disease Journal. 2010 Jun; 29(6):559-61. 